\title{
A Molecular Communications Framework for Understanding the Floral Transition
}

\author{
Maurizio Magarini \\ Politecnico di Milano \\ Milan, Italy \\ maurizio.magarini@polimi.it \\ Maximilian Schäfer \\ University of Erlangen-Nürnberg \\ Erlangen, Germany \\ max.schaefer@fau.de
}

\author{
Richard J. Morris \\ John Innes Centre \\ Norwich, UK \\ richard.morris@jic.ac.uk \\ George W. Bassel
The University of Warwick \\ George W. Bassel
The University of Warwick \\ Coventry, UK \\ george.bassel@warwick.ac.uk \\ Werner Haselmayr \\ Johannes Kepler University \\ Linz, Austria \\ werner.haselmayr@jku.at
}

\author{
Francesca Ratti \\ Politecnico di Milano \\ Milan, Italy \\ francesca.ratti@polimi.it
}

Hamdan Awan

Waterford Institute of Technology

Waterford, Ireland

hawan@tssg.org

\begin{abstract}
ACM Reference Format:
Maurizio Magarini, Richard J. Morris, Francesca Ratti, Maximilian Schäfer, George W. Bassel, Hamdan Awan, and Werner Haselmayr. 2020. A Molecular Communications Framework for Understanding the Floral Transition. In The Seventh Annual ACM International Conference on Nanoscale Computing and Communication (NANOCOM '20), September 23-25, 2020, Virtual Event, USA. ACM, New York, NY, USA, 2 pages. https://doi.org/10.1145/3411295.3411301
\end{abstract}

\section{INTRODUCTION}

With no option to run away, plants' survival builds on their capacity to respond to challenges from their environment. Plants monitor their surroundings and plastically adjust their metabolism, growth and development to acclimatise and defend themselves [1]. In particular, the timing of events such as germination, flowering, pollination and seed development are key adaptive traits [2, 3]. Plants go to great lengths to get the timing of these developmental transitions coordinated with their environmental conditions [4].

The transition of the vegetative to reproductive phase is an important developmental decision that is crucial for plant fitness [5]. Well over a hundred genes have been identified in this transition in Arabidopsis, demonstrating the importance of this process to the plant. Unsurprisingly, flowering time has received a great deal of attention from plant biologists and many genetic pathways are now well documented [6, 7].

Fig. 1(a) gives a conceptual overview of the process. Plants measure day length and light intensity in their leaves [8-10]. Day length is determined by linking light detection to plant's internal circadian clock and producing a mobile protein called Flowering locus T (FT), now commonly thought to be the flowering signal florigen. FT is exported from companion cells into the phloem stream where it is transported through fluid flow to the shoot apical meristem [8].

Permission to make digital or hard copies of all or part of this work for personal or classroom use is granted without fee provided that copies are not made or distributed for profit or commercial advantage and that copies bear this notice and the full citation on the first page. Copyrights for components of this work owned by others than ACM must be honored. Abstracting with credit is permitted. To copy otherwise, or republish, to post on servers or to redistribute to lists, requires prior specific permission and/or a fee. Request permissions from permissions@acm.org.

NANOCOM '20, September 23-25, 2020, Virtual Event, USA

(C) 2020 Association for Computing Machinery.

ACM ISBN 978-1-4503-8083-6/20/09...\$15.00

https://doi.org/10.1145/3411295.3411301

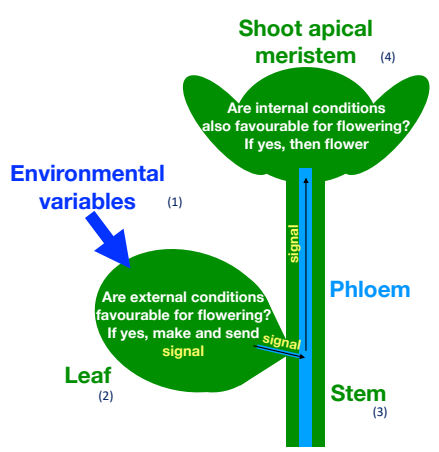

(a)

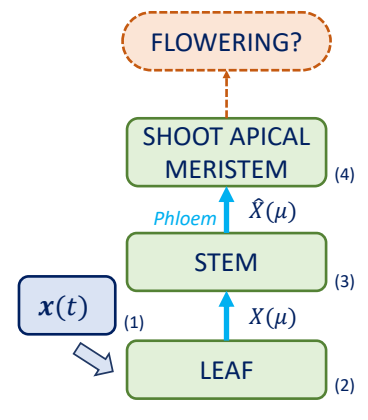

(b)
Figure 1: How plants measure environmental conditions to determine when to enter their reproductive phase (flowering) can be viewed as an application of molecular communication (MC) theory. (a): Main biological steps in the transition from vegetative growth to flowering. Gene regulatory networks carry out signal processing and signal integration. (b): Processing blocks of the floral transition. The corresponding MC processes will be used to model the function of gene regulatory networks active in leaf and apex tissue.

Here, FT is unloaded and initiates a response via an integrative gene regulatory that integrate the signal to control when to flower [11]

\section{ROLE OF MOLECULAR COMMUNICATIONS IN PLANTS}

Molecular communication (MC) plays a vital role in different functions of plants such as growth, self regulation and response to different harmful stress. As shown in recent work [12], the external stress signals such as light, temperature or pressure result in Action Potential (AP) signal in the leaf cells of plants. This AP signal can trigger the release of information carrying molecules which propagate to the neighbouring cells and result in a collective action taken by the leaf of plant such as increase or decrease in growth. The mutual information of the MC system has a direct impact on this action of the plant in response to external stress. Like wise on a much larger scale (compared to leaves) the MC phenomena plays an important role in the everyday functions of plants. For example one of such important role is to control the flowering time in plants. 
As flowering is a key characteristic of any plant, the accuracy and optimal timing needs to be carefully chosen. In this work, we point out a number of possible studies which need to be explored in order to develop a better understanding on how plants determine the flowering time.

\section{AN MC FRAMEWORK FOR UNDERSTANDING FLOWERING TIME}

Here, we propose a functional model based on ideas from MC for understanding how plants determine when to flower. Placing the wealth of genetic and physiological information about the floral transition within the framework of communication theory involves determining the encoding and decoding functions, dissecting the mode of the transmission, and placing these events within the geometry (and connecting topology) of the plant. The dissection of these processes requires an iterative and interdisciplinary approach that brings together expertise from the fields of plant biology, cellular topology, gene regulatory networks, system modelling, microfluidics. The availability of a functional model can help in answering to critical challenges such as how flowering would change with climate changes or which crop varieties do better under different climate scenarios.

Before discussing modelling approaches and challenges in Sec. 4, the individual processing blocks in Fig. 1(b) are defined:

(1) The external influences that are measured by the leaf, are defined by the vector of source signals

$$
x(t)=\left[\begin{array}{c}
I_{\lambda}(t) \\
T(t) \\
\vdots
\end{array}\right] \quad \text { Light intensity }
$$

Among others, the perceived intensity of light $I_{\lambda}$ at different wavelengths $\lambda$ and the temperature $T(t)$ perceived by the leaf during the day.

(2) Based on the perceived signals in $x$ the leaf produces the protein FT. Therefore, the leaf acts as encoder which transforms the continuous source signal $x(t)$ into a "discrete" signal $X(\mu)$ that represents individual FTs.

(3) The stem can be interpreted as the transmission channel that transports the FT into the apical meristem. Similarly to classical communication systems, the channel may introduce supporting or interfering effects on the transmitted signal. Therefore, the signal after the channel is denoted by $\hat{X}(\mu)$.

(4) The apical meristem is interpreted as the decoder of the system where the FT is unloaded and used to determine when to flower.

\section{CHALLENGES AND DISCUSSION}

With reference to Fig. 1(b), suitable models need to be developed for the source signal, encoder, channel, and decoder sub-systems. In analogy to analog and digital communication systems, the goal is to give a statistical description of all the involved signals (e.g., through mutual information), which is the basis for the evaluation of performance in terms of information transfer rate considering the probabilistic nature of the source and decoding processes. For this reason, most of the ideas discussed in what follows are directly inspired by the modulation and detection theory in communication systems and also from some microfluidic aspects of transport within the phloem. More detailed investigations are planned for future work.
Source Considering the periodicity of light during the day, a possible description of the source signal can be cast in the framework of a classical modulation scheme, where the parameters of a periodic wave, i.e. the carrier, are changed according to intensity and spatial distribution of light, duration of exposure, time of the day, and spectral composition. Considering the alternation of daylight and darkness, a straightforward characterization could be that of associating their relative duration to the duty-cycle of a 24-hours periodic wave. With this interpretation, the duration of daylight controls the width of an analog pulse-width modulated (PWM) waveform. The width varies with a yearly periodicity and depends on many factors such as geographical latitude and altitude, as well as seasons. Random variations of the width can be introduced to describe the random production of FT.

Encoder The encoder, which receives at the input the PWM waveform and produces a pulsed signal at its output, plays a role similar to that of an analog-to-digital converter. The resulting pulsed waveform at the output is characterized by spikes whose width changes according to the measure of the duty cycle of the input PWM waveform. The variable width can be thought as obtained at the output of a comparator-like electronic device that maintains a constant signal value for a time proportional to the duration of the duty-cycle, when this latter is larger than a reference, possibly variable, level. Channel The transport process associated with the phloem stream can be modeled as microfluidic channel in a bounded environment. However, starting from this initial and intuitive characterization, a more in depth study of effects introduced by phloem stream on parameters like drift, diffusion, etc. needs to be developed.

Decoder A formulation of flowering time can be cast as a statistical hypothesis testing problem. This can be directly related to the processing of FTs done by gene regulatory networks, which are used to model flowering time control and induction. A simple threshold detector can be considered whose threshold can be designed on the basis of the statistical characterization of the received after the low-pass filtered signal at the output of the pistil. However, more sophisticated detectors can be developed to integrate over signal history and consider the realistic situation where there is memory.

\section{REFERENCES}

[1] C. Turnbull. Long-distance regulation of flowering time. Fournal of experimental botany, 62(13):4399-413, Aug. 2011.

[2] A. Mouradov, F. Cremer, and G. Coupland. Control of flowering time interacting pathways as a basis for diversity. Plant Cell, pages 111-131, 2002.

[3] C. Jung and A. E. Müller. Flowering time control and applications in plant breeding. Trends in plant science, 14(10):563-73, Oct. 2009.

[4] M. Koornneef, C. Alonso-Blanco, A. J. Peeters, and W. Soppe. Genetic Control of Flowering Time in Arabidopsis. Annu. Rev. Plant Physiol. Plant Mol. Biol., 49:345-370, Jun. 1998.

[5] G. G. Simpson and C. Dean. Arabidopsis, the Rosetta stone of flowering time? Science (New York, N.Y.), 296(5566):285-9, Apr. 2002.

[6] Y. Komeda. Genetic regulation of time to flower in Arabidopsis thaliana. Annu. Rev. Plant Biol., 55:521-35, Jan. 2004.

[7] F. Andrés and G. Coupland. The genetic basis of flowering responses to seasonal cues. Nat. Rev. Genet., 13(9):627-39, Sep. 2012

[8] F. Turck, F. Fornara, and G. Coupland. Regulation and identity of florigen: FLOWERING LOCUS T moves center stage. Annual review of plant biology, 59:573-94, Jan. 2008.

[9] Y. H. Song, S. Ito, and T. Imaizumi. Flowering time regulation: photoperiod- and temperature-sensing in leaves. Trends in plant science, 18(10):575-83, Oct. 2013.

[10] D. D. Seaton, R. W. Smith, Y. H. Song, D. R. MacGregor, K. Stewart, G. Steel, J. Foreman, S. Penfield, T. Imaizumi, A. J. Millar, and K. J. Halliday. Linked circadian outputs control elongation growth and flowering in response to photoperiod and temperature. Molecular systems biology, 11(1):776, Jan. 2015.

[11] K. E. Jaeger, N. Pullen, S. Lamzin, R. J. Morris, and P. A. Wigge. Interlocking feedback loops govern the dynamic behavior of the floral transition in Arabidopsis. Plant cell, 25(3):820-33, Mar. 2013.

[12] H. Awan, K. Zeid, R. S. Adve, N. Wallbridge, C. Plummer, and A. W. Eckford. Communication in plants: Comparison of multiple action potential and mechanosensitive signals with experiments. IEEE T. Nanobiosci., 2019. 